\title{
GLOBAL DYNAMICS OF THE BENOÎT SYSTEM
}

\author{
MAURÍCIO FIRMINO SILVA LIMA ${ }^{1}$ AND JAUME LLIBRE ${ }^{2}$
}

\begin{abstract}
In this paper we work with a two-degree polynomial differential system in $\mathbb{R}^{3}$ related with the canard phenomena. We show that this system is completely integrable, and we provide its global phase portrait in the Poincaré ball using the Poincaré-Lyapunov compactification.
\end{abstract}

\section{INTRODUCTION}

We recall that the relevant canard phenomena were discorever dy the French mathematicians E. Benoît, J.C. Callot, F. Diener and M. Diener [4] in 1981 when they studied the 2-dimensional van der Pol oscillator. These canard phenomena are generically persistent under small parameter changes in singular perturbed differential systems with two or more slow variables and one fast variable, see [2] and also [8], [9], [3] and [10].

In the paper of Benoît [2] of the year 1983 a slow-fast system in $\mathbb{R}^{3}$ was studied with 2 slow and 1 fast variable. This paper becomes seminal because it leads to the well-known mixed-mode oscillations investigated up to now. Choosing appropriate weights, the main part of the vector field is the quasi-homogeneous part of degree 2 that exhibits the more interesting dynamics. It is precisely this quasi-homogeneous part that we choose as the differential system to study in this paper. A reason for looking at this quasi-homogeneous part comes from the geometric approach of singular perturbations, due to Wechselberger and Szmolyan (see [10] and [9]), where a blow-up (rescaling) is used and all terms of the vector field that do not belong to this quasi-homogeneous part become of higher order after the rescaling. Another reason is that up to know no body described the global dynamics of this interesting system.

In short we consider the 3 -dimensional polynomial differential system in $\mathbb{R}^{3}$ given by

$$
\begin{aligned}
& X^{\prime}=-2 Y, \\
& Y^{\prime}=1, \\
& Z^{\prime}=-\frac{X+Z^{2}}{\varepsilon} .
\end{aligned}
$$

Here $\varepsilon$ is a nonzero real parameter and the prime denotes the derivative with respect to the independent variable $T$ that we call the time. This system in this paper will be called the Benoît system.

The objective of this paper is to describe the global dynamics of the Benoit system (1). For doing this, first we rescale all the variables and we eliminate the

2010 Mathematics Subject Classification. 34A36, 34A60, 34C25, 34C37.

Key words and phrases. equilibrium point, first integral, invariant manifolds, homoclinic orbit, heteroclinic orbit, Poincaré compactification, Poincaré sphere, Benoît system. 
redundant parameter $\varepsilon$. After we will use the Poincaré compactification of $\mathbb{R}^{3}$. This compactification identifies $\mathbb{R}^{3}$ with the interior of the unit ball in $\mathbb{R}^{3}$ and the infinity of $\mathbb{R}^{3}$ with the boundary $\mathbb{S}^{2}$ of that ball, and the Benoît system is analytically extended to the closed ball which is called the Poincaré ball. This extended flow has the sphere $\mathbb{S}^{2}$ invariant. We shall describe the phase of the Benoit system in the Poincaré sphere using the Poincaré-Lyapunov compactification, see for more details section 2 .

All the results that we shall present and prove will be for $\varepsilon>0$. In fact it is not difficult to see that the dynamics for the case $\varepsilon<0$ can be studied in a similar way to the case $\varepsilon>0$. In fact, it is easy to check that the phase portrait of the Benoît system in the Poincaré ball for $\varepsilon=\varepsilon^{*}<0$ are the same that for $\varepsilon=-\varepsilon^{*}>0$ but changing the sign of the $z$ coordinate.

Doing the rescaling $(X, Y, Z, T) \rightarrow(x, y, z, t)$ defined by $X=\varepsilon x, Y=\varepsilon^{1 / 2} y$, $Z=\varepsilon^{1 / 2} z$ and $T=\varepsilon^{1 / 2} t$ the differential system (1) becomes

$$
\begin{array}{ll}
\dot{x}=-2 y & =P_{1}(x, y, z), \\
\dot{y}=1 & =P_{2}(x, y, z), \\
\dot{z}=-x-z^{2} & =P_{3}(x, y, z) .
\end{array}
$$

The strategy for providing the phase portrait in the Poincaré ball of the Benoit system is the following. First we study the Benoit system (2) at infinity that corresponds to analyze the extended Benoit system restricted to the boundary $\mathbb{S}^{2}$. Second we state two independent first integrals for the Benoît system and we also provide the explicit solutions of the Benoit system in function of the new time $t$. Third we describe its global dynamics in the Poincaré ball. We shall see that all the orbits of the Benoît system will have their $\alpha$ - and $\omega$-limit at infinity.

The main results of this paper are the following.

Proposition 1. The extended Benôt system at infinity has as equilibrium points, at the Poincaré-Lyapunov sphere, the end of the $x$-axis (which are saddle-nodes) and z-axis (which are hyperbolic nodes). Moreover the phase portrait of the extended Benoît system on the Poincaré-Lyapunov sphere is represented, in Figure 1.
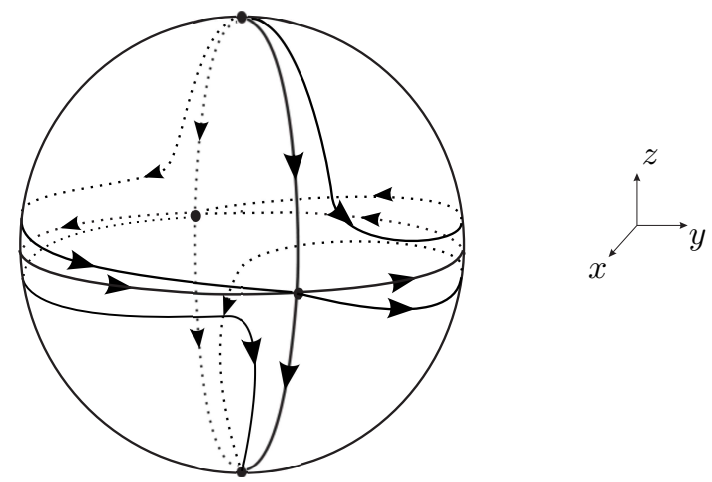

Figure 1. Phase portrait of the Benoît system at the PoincaréLyapunov sphere at infinity.

Proposition 1 is proved in section 3 . 
Proposition 2. The Benoit system is completely integrable having the following two independent first integrals

$$
H_{1}=x+y^{2}
$$

and $\mathrm{H}_{2}$ is equal to

$$
\begin{aligned}
& {\left[8 W_{1}\left(\frac{y^{2}}{4}+\frac{x}{4}, \frac{1}{4}, y^{2}\right) W_{1}\left(\frac{y^{2}}{4}+\frac{x}{4}+1, \frac{1}{4}, y^{2}\right)-2 W_{1}\left(\frac{y^{2}}{4}+\frac{x}{4}, \frac{1}{4}, y^{2}\right)^{2} y^{2}+W_{1}\left(\frac{y^{2}}{4}+\frac{x}{4}, \frac{1}{4}, y^{2}\right)^{2} y^{4}\right.} \\
& +2 W_{1}\left(\frac{y^{2}}{4}+\frac{x}{4}, \frac{1}{4}, y^{2}\right)^{2} x+W_{1}\left(\frac{y^{2}}{4}+\frac{x}{4}, \frac{1}{4}, y^{2}\right)^{2} x^{2}-2 W_{1}\left(\frac{y^{2}}{4}+\frac{x}{4}, \frac{1}{4}, y^{2}\right)^{2} y^{2} x \\
& -8 W_{1}\left(\frac{y^{2}}{4}+\frac{x}{4}, \frac{1}{4}, y^{2}\right) y^{2} W_{1}\left(\frac{y^{2}}{4}+\frac{x}{4}+1, \frac{1}{4}, y^{2}\right)+8 W_{1}\left(\frac{y^{2}}{4}+\frac{x}{4}, \frac{1}{4}, y^{2}\right) W_{1}\left(\frac{y^{2}}{4}+\frac{x}{4}+1, \frac{1}{4}, y^{2}\right) x \\
& \left.+16 W_{1}\left(\frac{y^{2}}{4}+\frac{x}{4}+1, \frac{1}{4}, y^{2}\right)^{2}+W_{1}\left(\frac{y^{2}}{4}+\frac{x}{4}, \frac{1}{4}, y^{2}\right)^{2}\right]^{\frac{1}{2}} z^{2} \\
& +\left[2 W_{2}\left(\frac{y^{2}}{4}+\frac{x}{4}, \frac{1}{4}, y^{2}\right) W_{1}\left(\frac{y^{2}}{4}+\frac{x}{4}, \frac{1}{4}, y^{2}\right)-8 W_{2}\left(\frac{y^{2}}{4}+\frac{x}{4}+1, \frac{1}{4}, y^{2}\right) W_{1}\left(\frac{y^{2}}{4}+\frac{x}{4}+1, \frac{1}{4}, y^{2}\right) y^{2}\right. \\
& +2 z y W_{1}\left(\frac{y^{2}}{4}+\frac{x}{4}, \frac{1}{4}, y^{2}\right)+2 W_{2}\left(\frac{y^{2}}{4}+\frac{x}{4}, \frac{1}{4}, y^{2}\right) y^{4} W_{1}\left(\frac{y^{2}}{4}+\frac{x}{4}, \frac{1}{4}, y^{2}\right) \\
& 8 W_{2}\left(\frac{y^{2}}{4}+\frac{x}{4}, \frac{1}{4}, y^{2}\right) y^{2} W_{1}\left(\frac{y^{2}}{4}+\frac{x}{4}+1, \frac{1}{4}, y^{2}\right)+8 W_{2}\left(\frac{y^{2}}{4}+\frac{x}{4}, \frac{1}{4}, y^{2}\right) W_{1}\left(\frac{y^{2}}{4}+\frac{x}{4}+1, \frac{1}{4}, y^{2}\right) \\
& -6 W_{2}\left(\frac{y^{2}}{4}+\frac{x}{4}+1, \frac{1}{4}, y^{2}\right) W_{1}\left(\frac{y^{2}}{4}+\frac{x}{4}, \frac{1}{4}, y^{2}\right)-4 W_{2}\left(\frac{y^{2}}{4}+\frac{x}{4}, \frac{1}{4}, y^{2}\right) W_{1}\left(\frac{y^{2}}{4}+\frac{x}{4}, \frac{1}{4}, y^{2}\right) y^{2} x \\
& -8 x W_{1}\left(\frac{y^{2}}{4}+\frac{x}{4}+1, \frac{1}{4}, y^{2}\right) W_{2}\left(\frac{y^{2}}{4}+\frac{x}{4}+1, \frac{1}{4}, y^{2}\right)+2 W_{2}\left(\frac{y^{2}}{4}+\frac{x}{4}, \frac{1}{4}, y^{2}\right) W_{1}\left(\frac{y^{2}}{4}+\frac{x}{4}, \frac{1}{4}, y^{2}\right) x^{2} \\
& +8 W_{2}\left(\frac{y^{2}}{4}+\frac{x}{4}, \frac{1}{4}, y^{2}\right) W_{1}\left(\frac{y^{2}}{4}+\frac{x}{4}+1, \frac{1}{4}, y^{2}\right) x-4 W_{2}\left(\frac{y^{2}}{4}+\frac{x}{4}, \frac{1}{4}, y^{2}\right) W_{1}\left(\frac{y^{2}}{4}+\frac{x}{4}, \frac{1}{4}, y^{2}\right) y^{2} \\
& -8 W_{2}\left(\frac{y^{2}}{4}+\frac{x}{4}+1, \frac{1}{4}, y^{2}\right) W_{1}\left(\frac{y^{2}}{4}+\frac{x}{4}, \frac{1}{4}, y^{2}\right) x+4 W_{2}\left(\frac{y^{2}}{4}+\frac{x}{4}+1, \frac{1}{4}, y^{2}\right) W_{1}\left(\frac{y^{2}}{4}+\frac{x}{4}, \frac{1}{4}, y^{2}\right) y^{2} \\
& -24 W_{1}\left(\frac{y^{2}}{4}+\frac{x}{4}+1, \frac{1}{4}, y^{2}\right) W_{2}\left(\frac{y^{2}}{4}+\frac{x}{4}+1, \frac{1}{4}, y^{2}\right) \\
& +2 W_{2}\left(\frac{y^{2}}{4}+\frac{x}{4}+1, \frac{1}{4}, y^{2}\right) W_{1}\left(\frac{y^{2}}{4}+\frac{x}{4}, \frac{1}{4}, y^{2}\right) y^{4}+4 W_{2}\left(\frac{y^{2}}{4}+\frac{x}{4}, \frac{1}{4}, y^{2}\right) W_{1}\left(\frac{y^{2}}{4}+\frac{x}{4}, \frac{1}{4}, y^{2}\right) x \\
& \left.-2 W_{2}\left(\frac{y^{2}}{4}+\frac{x}{4}+1, \frac{1}{4}, y^{2}\right) W_{1}\left(\frac{y^{2}}{4}+\frac{x}{4}, \frac{1}{4}, y^{2}\right) x^{2}\right] z \\
& +9 W_{2}\left(\frac{y^{2}}{4}+\frac{x}{4}+1, \frac{1}{4}, y^{2}\right)^{2}+6 W_{2}\left(\frac{y^{2}}{4}+\frac{x}{4}+1, \frac{1}{4}, y^{2}\right)^{2} y^{2}+6 W_{2}\left(\frac{y^{2}}{4}+\frac{x}{4}+1, \frac{1}{4}, y^{2}\right)^{2} x \\
& +W_{2}\left(\frac{y^{2}}{4}+\frac{x}{4}+1, \frac{1}{4}, y^{2}\right)^{2} y^{4}+W_{2}\left(\frac{y^{2}}{4}+\frac{x}{4}+1, \frac{1}{4}, y^{2}\right)^{2} x^{2}+2 W_{2}\left(\frac{y^{2}}{4}+\frac{x}{4}+1, \frac{1}{4}, y^{2}\right)^{2} y^{2} x \\
& +4 W_{2}\left(\frac{y^{2}}{4}+\frac{x}{4}, \frac{1}{4}, y^{2}\right) y^{2} W_{2}\left(\frac{y^{2}}{4}+\frac{x}{4}+1, \frac{1}{4}, y^{2}\right)+2 W_{2}\left(\frac{y^{2}}{4}+\frac{x}{4}, \frac{1}{4}, y^{2}\right) y^{4} W_{2}\left(\frac{y^{2}}{4}+\frac{x}{4}+1, \frac{1}{4}, y^{2}\right) \\
& -8 W_{2}\left(\frac{y^{2}}{4}+\frac{x}{4}, \frac{1}{4}, y^{2}\right) W_{2}\left(\frac{y^{2}}{4}+\frac{x}{4}+1, \frac{1}{4}, y^{2}\right) x \\
& -2 W_{2}\left(\frac{y^{2}}{4}+\frac{x}{4}, \frac{1}{4}, y^{2}\right) W_{2}\left(\frac{y^{2}}{4}+\frac{x}{4}+1, \frac{1}{4}, y^{2}\right) x^{2}+2 z y W_{2}\left(\frac{y^{2}}{4}+\frac{x}{4}, \frac{1}{4}, y^{2}\right) \\
& -2 W_{2}\left(\frac{y^{2}}{4}+\frac{x}{4}, \frac{1}{4}, y^{2}\right)^{2} y^{2} x+W_{2}\left(\frac{y^{2}}{4}+\frac{x}{4}, \frac{1}{4}, y^{2}\right)^{2} y^{4}+2 W_{2}\left(\frac{y^{2}}{4}+\frac{x}{4}, \frac{1}{4}, y^{2}\right)^{2} x \\
& W_{2}\left(\frac{y^{2}}{4}+\frac{x}{4}, \frac{1}{4}, y^{2}\right)^{2} x^{2}-2 W_{2}\left(\frac{y^{2}}{4}+\frac{x}{4}, \frac{1}{4}, y^{2}\right)^{2} y^{2}+W_{2}\left(\frac{y^{2}}{4}+\frac{x}{4}, \frac{1}{4}, y^{2}\right)^{2} \\
& -6 W_{2}\left(\frac{y^{2}}{4}+\frac{x}{4}, \frac{1}{4}, y^{2}\right) W_{2}\left(\frac{y^{2}}{4}+\frac{x}{4}+1, \frac{1}{4}, y^{2}\right) \text {. }
\end{aligned}
$$

The first integral $H_{1}$ is well known (see [2]), but as far as we know the second integral $\mathrm{H}_{2}$ is new.

Here $W_{1}(\mu, \nu, z)$ and $W_{2}(\mu, \nu, z)$ represent the Whittaker functions that are two independent solutions of the differential equation

$$
\ddot{y}+\left(-\frac{1}{4}+\frac{\mu}{\nu}+\frac{\frac{1}{4}-\nu^{2}}{z^{2}}\right) y=0 .
$$

They can be defined in terms of the Hypergeometric and Kummer functions in the following way:

$$
W_{2}(\mu, \nu, z)=e^{-z / 2} e^{1 / 2+\nu} H\left(\left[\frac{1}{2}+\nu-\mu\right],[1+2 \nu], z\right)
$$


and

$$
W_{1}(\mu, \nu, z)=e^{-z / 2} e^{1 / 2+\nu} K\left(\frac{1}{2}+\nu-\mu, 1+2 \nu, z\right) .
$$

For more details in these functions see for instance [1].

Proposition 3. The solution $(x(t), y(t), z(t))$ of the Benoit system satisfying $(x(0)$, $y(0), z(0))=\left(x_{0}, y_{0}, z_{0}\right) \in \mathbb{R}^{3}$ is given by $x(t)=-t^{2}-y_{0} t+x_{0}, y(t)=t+y_{0}$, and $z(t)$ is equal to

$$
\begin{aligned}
& -\frac{1}{3 d}\left\{-3 H\left(\left[\frac{5}{4}-\frac{x_{0}}{4}-\frac{y_{0}^{2}}{4}\right],\left[\frac{3}{2}\right],\left(t+y_{0}\right)^{2}\right) t\right. \\
& -3 H\left(\left[\frac{5}{4}-\frac{x_{0}}{4}-\frac{y_{0}^{2}}{4}\right],\left[\frac{3}{2}\right],\left(t+y_{0}\right)^{2}\right) y_{0}+3 H\left(\left[\frac{5}{4}-\frac{x_{0}}{4}-\frac{y_{0}^{2}}{4}\right],\left[\frac{3}{2}\right],\left(t+y_{0}\right)^{2}\right) x_{0} t \\
& +3 H\left(\left[\frac{5}{4}-\frac{x_{0}}{4}-\frac{y_{0}^{2}}{4}\right],\left[\frac{3}{2}\right],\left(t+y_{0}\right)^{2}\right) x_{0} y_{0}+3 H\left(\left[\frac{5}{4}-\frac{x_{0}}{4}-\frac{y_{0}^{2}}{4}\right],\left[\frac{3}{2}\right],\left(t+y_{0}\right)^{2}\right) y_{0}^{2} t \\
& +3 H\left(\left[\frac{5}{4}-\frac{x_{0}}{4}-\frac{y_{0}^{2}}{4}\right],\left[\frac{3}{2}\right],\left(t+y_{0}\right)^{2}\right) y_{0}^{3}+3 H\left(\left[\frac{1}{4}-\frac{x_{0}}{4}-\frac{y_{0}^{2}}{4}\right],\left[\frac{1}{2}\right],\left(t+y_{0}\right)^{2}\right) t \\
& +3 H\left(\left[\frac{1}{4}-\frac{x_{0}}{4}-\frac{y_{0}^{2}}{4}\right],\left[\frac{1}{2}\right],\left(t+y_{0}\right)^{2}\right) y_{0}-3 z_{0} H\left(\left[\frac{7}{4}-\frac{x_{0}}{4}-\frac{y_{0}^{2}}{4}\right],\left[\frac{5}{2}\right],\left(t+y_{0}\right)^{2}\right) t^{2} \\
& -6 z_{0} H\left(\left[\frac{7}{4}-\frac{x_{0}}{4}-\frac{y_{0}^{2}}{4}\right],\left[\frac{5}{2}\right],\left(t+y_{0}\right)^{2}\right) y_{0} t-3 z_{0} H\left(\left[\frac{7}{4}-\frac{x_{0}}{4}-\frac{y_{0}^{2}}{4}\right],\left[\frac{5}{2}\right],\left(t+y_{0}\right)^{2}\right) y_{0}^{2} \\
& +z_{0} H\left(\left[\frac{7}{4}-\frac{x_{0}}{4}-\frac{y_{0}^{2}}{4}\right],\left[\frac{5}{2}\right],\left(t+y_{0}\right)^{2}\right) t^{2} x_{0}+2 z_{0} H\left(\left[\frac{7}{4}-\frac{x_{0}}{4}-\frac{y_{0}^{2}}{4}\right],\left[\frac{5}{2}\right],\left(t+y_{0}\right)^{2}\right) y_{0} t x_{0} \\
& +z_{0} H\left(\left[\frac{7}{4}-\frac{x_{0}}{4}-\frac{y_{0}^{2}}{4}\right],\left[\frac{5}{2}\right],\left(t+y_{0}\right)^{2}\right) y_{0}^{2} x_{0}+z_{0} H\left(\left[\frac{7}{4}-\frac{x_{0}}{4}-\frac{y_{0}^{2}}{4}\right],\left[\frac{5}{2}\right],\left(t+y_{0}\right)^{2}\right) y_{0}^{2} t^{2} \\
& +2 z_{0} H\left(\left[\frac{7}{4}-\frac{x_{0}}{4}-\frac{y_{0}^{2}}{4}\right],\left[\frac{5}{2}\right],\left(t+y_{0}\right)^{2}\right) y_{0}^{3} t+z_{0} H\left(\left[\frac{7}{4}-\frac{x_{0}}{4}-\frac{y_{0}^{2}}{4}\right],\left[\frac{5}{2}\right],\left(t+y_{0}\right)^{2}\right) y_{0}^{4} \\
& -3 z_{0} H\left(\left[\frac{3}{4}-\frac{x_{0}}{4}-\frac{y_{0}^{2}}{4}\right],\left[\frac{3}{2}\right],\left(t+y_{0}\right)^{2}\right)+3 z_{0} H\left(\left[\frac{3}{4}-\frac{x_{0}}{4}-\frac{y_{0}^{2}}{4}\right],\left[\frac{3}{2}\right],\left(t+y_{0}\right)^{2}\right) t^{2} \\
& \left.+6 z_{0} H\left(\left[\frac{3}{4}-\frac{1}{4}-\frac{y_{0}^{2}}{4}\right],\left[\frac{3}{2}\right],\left(t+y_{0}\right)^{2}\right) y_{0} t+3 z_{0} H\left(\left[\frac{3}{4}-\frac{x_{0}}{4}-\frac{y_{0}^{2}}{4}\right],\left[\frac{3}{2}\right],\left(t+y_{0}\right)^{2}\right) y_{0}^{2}\right\},
\end{aligned}
$$

where

$$
\begin{aligned}
d= & z_{0} H\left(\left[\frac{3}{4}-\frac{x_{0}}{4}-\frac{y_{0}^{2}}{4}\right],\left[\frac{3}{2}\right],\left(t+y_{0}\right)^{2}\right) t+z_{0} H\left(\left[\frac{3}{4}-\frac{x_{0}}{4}-\frac{y_{0}^{2}}{4}\right],\left[\frac{3}{2}\right],\left(t+y_{0}\right)^{2}\right) y_{0} \\
& +H\left(\left[\frac{1}{4}-\frac{x_{0}}{4}-\frac{y_{0}^{2}}{4}\right],\left[\frac{1}{2}\right],\left(t+y_{0}\right)^{2}\right) .
\end{aligned}
$$

Corollary 4. All the orbits of the Benoit system are unbounded in forward and backward time.

Theorem 5. The possible orbits of the Benoit system restricted to the level $H_{1}=h_{1}$ are of three types:

(i) Disconnecting orbits: homoclinic orbits to the equilibrium point at infinity $(-1,0,0) \in \mathbb{S}^{2}$

(ii) Intermediate orbits: heteroclinic orbits going from the equilibrium $(0,0,1) \in$ $\mathbb{S}^{2}$ to the equilibrium $(-1,0,0) \in \mathbb{S}^{2}$, or going from the equilibrium $(-1,0,0) \in$ $\mathbb{S}^{2}$ to the equilibrium $(0,0,-1) \in \mathbb{S}^{2}$.

(iii) Passage orbits: heteroclinic orbits going from the equilibrium $(0,0,1) \in \mathbb{S}^{2}$ to the equilibrium $(0,0,-1) \in \mathbb{S}^{2}$.

The three possible configurations of the orbits are drawn in Figure 2.

Moreover all these three possible types appear in the Benoit system but in different levels of $H_{1}$.

Propositions 2 and 3 and Corollary 4 are proved in section 4, and Theorem 5 is proved in section 5 .

In the next section we present the Poincaré-Lyapunov compactification in $\mathbb{R}^{3}$. 


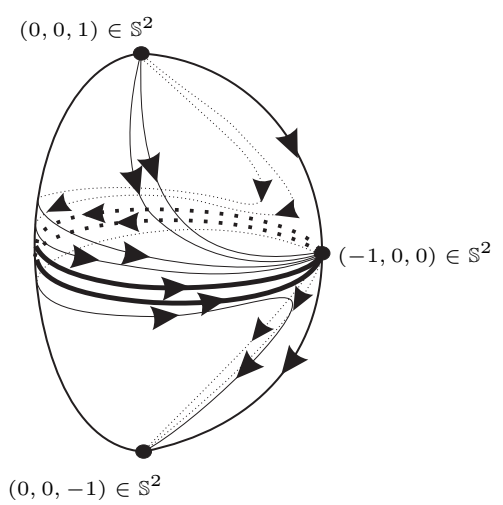

(a)

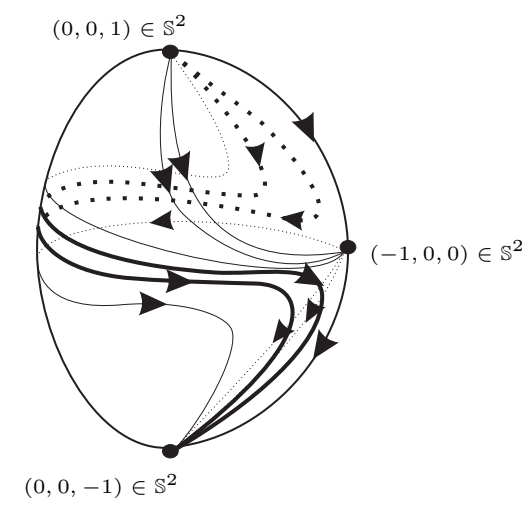

(c)

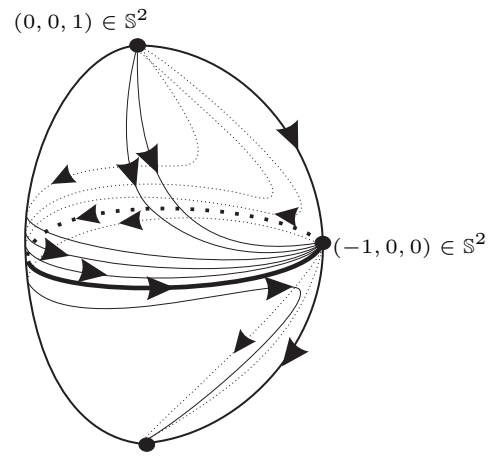

$(0,0,-1) \in \mathbb{S}^{2}$

(b)

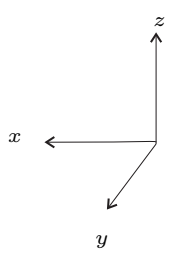

Figure 2. Possible phase portrait of the Benoît system restricted to the compactified level $H_{1}=h_{1}$. Picture (a) has a continuum of orbits of disconnecting type. Picture (b) has a unique orbit of disconnecting type. Picture (c) has no orbits of disconnecting type.

\section{Poincaré and Poincaré-Lyapunov compactification in $\mathbb{R}^{3}$}

A polynomial vector field $X$ in $\mathbb{R}^{3}$ can be extended to a unique analytic vector field on the sphere $\mathbb{S}^{2}$. The technique for doing such an extension is called the Poincaré compactification and allows to study a polynomial vector field in a neighborhood of infinity, which corresponds to the equator $\mathbb{S}^{2}$ of the sphere $\mathbb{S}^{3}$. Poincaré introduced this compactification for polynomial vector fields in $\mathbb{R}^{2}$. Its extension to $\mathbb{R}^{3}$ can be found in [5]. For completeness we will give here a small introduction on this technic as it appears in [5]. 
In $\mathbb{R}^{3}$ we consider the polynomial differential system

$$
\dot{x}=P_{1}(x, y, z), \quad \dot{y}=P_{2}(x, y, z), \quad \dot{z}=P_{3}(x, y, z),
$$

or equivalently its associated polynomial vector field $X=\left(P_{1}, P_{2}, P_{3}\right)$. The degree $m$ of $X$ is defined as $m=\max \left(\operatorname{deg}\left(P_{i}\right) ; i=1,2,3\right)$.

Let $\mathbb{S}^{3}=\left\{y=\left(y_{1}, y_{2}, y_{3}, y_{4}\right) \in \mathbb{R}^{4} ;\|y\|=1\right\}$ be the unit sphere in $\mathbb{R}^{4}, \mathbb{S}_{+}=\{y \in$ $\left.\mathbb{S}^{3} ; y_{4}>0\right\}$ and $\mathbb{S}_{-}=\left\{y \in \mathbb{S}^{3} ; y_{4}<0\right\}$ be the northern and southern hemispheres of $\mathbb{S}^{3}$ respectively. The tangent space to $\mathbb{S}^{3}$ at the point $y$ is denoted by $T_{y} \mathbb{S}^{3}$. Then the tangent plane

$$
T_{(0,0,0,1)} \mathbb{S}^{3}=\left\{\left(x_{1}, x_{2}, x_{3}, 1\right) \in \mathbb{R}^{4} ;\left(x_{1}, x_{2}, x_{3}\right) \in \mathbb{R}^{3}\right\}
$$

is identified with $\mathbb{R}^{3}$.

We consider the central projections $f_{+}: T_{(0,0,0,1)} \mathbb{S}^{3} \rightarrow \mathbb{S}_{+}$and $f_{-}: T_{(0,0,0,1)} \mathbb{S}^{3} \rightarrow$ $\mathbb{S}_{-}$defined by $f_{ \pm}(x)= \pm\left(x_{1}, x_{2}, x_{3}, 1\right) / \Delta x$, where $\Delta(x)=\left(1+\sum_{i=1}^{3} x_{i}^{2}\right)^{1 / 2}$. Through these central projection $\mathbb{R}^{3}$ is identified with the northern and southern hemispheres. The equator of $\mathbb{S}^{3}$ is $\mathbb{S}^{2}=\left\{y \in \mathbb{S}^{3} ; y_{4}=0\right\}$. Clearly $\mathbb{S}^{2}$ can be identified with the infinity of $\mathbb{R}^{3}$.

The maps $f_{+}$and $f_{-}$define two copies of $X$ on $\mathbb{S}^{3}$, one $D f_{+} \circ X$ in the northern hemisphere and the other $D f_{-} \circ X$ in the southern one. Denote by $\bar{X}$ the vector field on $\mathbb{S}^{3} \backslash \mathbb{S}^{2}$ which, restricted to $\mathbb{S}_{+}$coincides with $D f_{+} \circ X$ and restricted to $\mathbb{S}_{-}$coincides with $D f_{-} \circ X$.

The expression for $\bar{X}(y)$ on $\mathbb{S}_{+} \cup \mathbb{S}_{-}$is

$$
\bar{X}(y)=y_{4}\left(\begin{array}{ccc}
1-y_{1}^{2} & -y_{2} y_{1} & -y_{3} y_{1} \\
-y_{1} y_{2} & 1-y_{2}^{2} & -y_{3} y_{2} \\
-y_{1} y_{3} & -y_{2} y_{3} & 1-y_{3}^{2} \\
-y_{1} y_{4} & -y_{2} y_{4} & -y_{3} y_{4}
\end{array}\right)\left(\begin{array}{l}
P_{1} \\
P_{2} \\
P_{3}
\end{array}\right),
$$

where $P_{i}=P_{i}\left(y_{1} /\left|y_{4}\right|, y_{2} /\left|y_{4}\right|, y_{3} /\left|y_{4}\right|\right)$. Written in this way $\bar{X}(y)$ is a vector field in $\mathbb{R}^{4}$ tangent to the sphere $\mathbb{S}^{3}$.

Now we can analytically extend the vector field $\bar{X}(y)$ to the whole sphere $\mathbb{S}^{3}$ by considering $p(X)=y_{4}^{m-1} \bar{X}(y)$, where $m$ is the degree of $X$. This extended vector field $p(X)$ is called the Poincaré compactification of $X$ on $\mathbb{S}^{3}$.

As $\mathbb{S}^{3}$ is a differentiable manifold, in order to compute the expression for $p(X)$ we can consider the eight local charts $\left(\mathcal{U}_{i}, F_{i}\right),\left(\mathcal{V}_{i}, G_{i}\right)$, where $\mathcal{U}_{i}=\left\{y \in \mathbb{S}^{3} ; y_{i}>0\right\}$ and $\mathcal{V}_{i}=\left\{y \in \mathbb{S}^{3} ; y_{i}<0\right\}$ for $i=1,2,3,4$; the diffeomorphisms $F_{i}: \mathcal{U}_{i} \rightarrow \mathbb{R}^{3}$ and $G_{i}: \mathcal{V}_{i} \rightarrow \mathbb{R}^{3}$ for $i=1,2,3,4$ are the inverses of the central projections from the origin to the tangent planes at the points $( \pm 1,0,0,0),(0, \pm 1,0,0),(0,0, \pm 1,0)$ and $(0,0,0, \pm 1)$, respectively. Therefore $F_{i}(y)=-G_{i}(y)$. Now we do the computations on $\mathcal{U}_{1}$. Suppose that the origin $(0,0,0,0)$, the point $\left(y_{1}, y_{2}, y_{3}, y_{4}\right) \in \mathbb{S}^{3}$ and the point $\left(1, z_{1}, z_{2}, z_{3}\right)$ in the tangent plane to $\mathbb{S}^{3}$ at $(1,0,0,0)$ are collinear. Then we have $1 / y_{1}=z_{1} / y_{2}=z_{2} / y_{3}=z_{3} / y_{4}$, and consequently $F_{1}(y)=\left(y_{2} / y_{1}, y_{3} / y_{1}, y_{4} / y_{1}\right)=$ $\left(z_{1}, z_{2}, z_{3}\right)$ defines the coordinates on $\mathcal{U}_{1}$. As

$$
D F_{1}(y)=\left(\begin{array}{cccc}
-y_{2} / y_{1}^{2} & 1 / y_{1} & 0 & 0 \\
-y_{3} / y_{1}^{2} & 0 & 1 / y_{1} & 0 \\
-y_{4} / y_{1}^{2} & 0 & 0 & 1 / y_{1}
\end{array}\right)
$$


and $y_{4}^{m-1}=\left(z_{3} / \Delta z\right)^{m-1}$, the analytical vector field $p(X)$ becomes

$$
\frac{z_{3}^{m}}{(\Delta z)^{m-1}}\left(-z_{1} P_{1}+P_{2},-z_{2} P_{1}+P_{3},-z_{3} P_{1}\right)
$$

where $P_{i}=P_{i}\left(1 / z_{3}, z_{1} / z_{3}, z_{2} / z_{3}\right)$.

In a similar way we can deduce the expression of $p(X)$ in $\mathcal{U}_{2}$ and $\mathcal{U}_{3}$. These are

$$
\frac{z_{3}^{m}}{(\Delta z)^{m-1}}\left(-z_{1} P_{2}+P_{1},-z_{2} P_{2}+P_{3},-z_{3} P_{2}\right)
$$

where $P_{i}=P_{i}\left(z_{1} / z_{3}, 1 / z_{3}, z_{2} / z_{3}\right)$ in $\mathcal{U}_{2}$, and

$$
\frac{z_{3}^{m}}{(\Delta z)^{m-1}}\left(-z_{1} P_{3}+P_{1},-z_{2} P_{3}+P_{2},-z_{3} P_{3}\right)
$$

where $P_{i}=P_{i}\left(z_{1} / z_{3}, z_{2} / z_{3}, 1 / z_{3}\right)$ in $\mathcal{U}_{3}$.

The expression for $p(X)$ in $\mathcal{U}_{4}$ is $z_{3}^{m+1}\left(P_{1}, P_{2}, P_{3}\right)$ where $P_{i}=P_{i}\left(z_{1}, z_{2}, z_{3}\right)$. The expression for $p(X)$ in the local chart $\mathcal{V}_{i}$ is the same as in $\mathcal{U}_{i}$ multiplied by $(-1)^{m-1}$.

When we work with the expression of the compactified vector field $p(X)$ in the local charts we shall omit the factor $1 /(\Delta z)^{m-1}$. We can do that through a rescaling of the time variable.

We remark that all the points on the sphere at infinity $\mathbb{S}^{2}$ in the coordinates of any local chart have $z_{3}=0$.

In what follows we shall work with the orthogonal projection of $p(X)$ from the closed northern hemisphere to $y_{4}=0$, and we continue denoting this projected vector field by $p(X)$. Note that the projection of the closed northers hemisphere is a closed ball $B$ of radius one, whose interior is diffeomorphic to $\mathbb{R}^{3}$ and whose boundary $\mathbb{S}^{2}$ corresponds to the infinity of $\mathbb{R}^{3}$. Of course $p(X)$ is defined in the whole closed ball $B$ in such a way that the flow on the boundary is invariant. The new vector field on $B$ is called the Poincaré compactification of $X$, and $B$ is called the Poincaré ball, and $\partial B$ is called the Poincaré sphere at infinity.

The singularities at infinity in a Poincaré compactification can be quite degenerate, and sometimes there is the possibility of eliminate this degeneracy doing the so called Poincaré-Lyapunov compactification. This compactification results to be an extension of the Poincaré compactification presented before, in the sense that we no longer keep the construction homogeneous, but make it quasihomogeneous. In this context the construction is very similar to the one done in the Poincaré compactification case and the representation of the vector field in the local charts $\mathcal{U}_{i}$ and $\mathcal{V}_{i}$ are obtained in the following way: in the local chart $\mathcal{U}_{1}$ we consider the coordinates $z_{1}, z_{2}, z_{3}$ where $x=1 / z_{3}^{\alpha}, y=z_{1} / z_{3}^{\beta}, z=z_{2} / z_{3}^{\gamma}$. For the local charts $\mathcal{U}_{2}$ and $\mathcal{U}_{3}$ we take respectively $x=z_{1} / z_{3}^{\alpha}, y=1 / z_{3}^{\beta}, z=z_{2} / z_{3}^{\gamma}$ and $x=z_{1} / z_{3}^{\alpha}, y=z_{2} / z_{3}^{\beta}, z=1 / z_{3}^{\gamma}$. Observe that the difference between this new compactification and the Poincaré compactification is the presence of the constants $(\alpha, \beta, \gamma)$ for some well choosing positive integer numbers. The correspondent ball $B$ obtained in this extended version is called Poincaré-Lyapunov ball, and its frontier $\partial B$ (that corresponds to the infinity of $\mathbb{R}^{3}$ ) is called the Poincaré-Lyapunov sphere at infinity.

It is possible that for a system for which the usual Poincaré compactification has a non-elementary singular point at infinity, for well chosen $\alpha, \beta$ and $\gamma$, the PoincaréLyapunov compactification has only elementary singular points at infinity, or even 
no singular points at infinity at all. For more details in this construction see for instance chapters 5 and 9 of [6].

\section{Dynamical Behavior of Benoît System at infinity}

In this section we will provide the dynamics of the Benoît system at infinity. For doing this we will use the Poincaré-Lyapunov compactification with weights $(\alpha, \beta, \gamma)=(1,2,1)$.

In order to understand the behavior of the flow at the Poincaré-Lyapunov sphere we have to describe the flow in each one of the six local charts $\mathcal{U}_{i}$ and $\mathcal{V}_{i}$ for $i=1,2,3$.

3.1. Local chart $\mathcal{U}_{1}$. We call the coordinates in this chart by $\left(z_{1}, z_{2}, z_{3}\right)$. In order to obtain the expression of the vector field in this chart we must take $x=1 / z_{3}$, $y=z_{1} / z_{3}^{2}$ and $z=z_{2} / z_{3}$ and, after this change, multiply the resultant vector field by $z_{3}$. After doing this the Poincaré-Lyapunov compactification of the Benoît system in the local chart $\mathcal{U}_{1}$ is given by

$$
\dot{z}_{1}=4 z_{1}^{2}+z_{3}^{2}, \quad \dot{z}_{2}=-z_{3}-z_{2}^{2}+2 z_{1} z_{2}, \quad \dot{z}_{3}=2 z_{1} z_{3} .
$$

Restricting to the points of the Poincaré-Lyapunov sphere that correspond to the points at infinity $\left(z_{3}=0\right)$ the previous system becomes

$$
\dot{z}_{1}=4 z_{1}^{2}, \quad \dot{z}_{2}=-z_{2}\left(z_{2}-2 z_{1}\right), \quad \dot{z}_{3}=0 .
$$

The last equation reflects the fact that the infinity is invariant under the flow. From this system we see that the Benoît system has a unique equilibrium point at the origin of the chat $\mathcal{U}_{1}$. Moreover this equilibrium point is degenerate and, after performing a blowing-up, we have the phase portrait give in Figure 3.

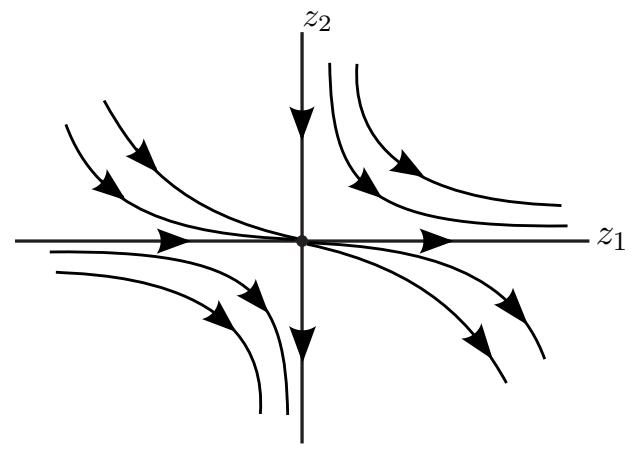

Figure 3 . The phase portrait in the local chart $\mathcal{U}_{1}$ at infinity.

Observe that the equilibrium point $(0,0,0)$ is located at the end of the positive part of the $x$-axis.

3.2. Local chart $\mathcal{U}_{2}$. In a similar way the Poincaré-Lyapunov compactification of the Benoît system in the local chart $\mathcal{U}_{2}$ is

$$
\dot{z}_{1}=-4-z_{1} z_{3}^{2}, \quad \dot{z}_{2}=-2 z_{1} z_{3}-z_{2}\left(2 z_{2}+z_{3}^{2}\right), \quad \dot{z}_{3}=-z_{3}^{4} .
$$

At infinity $\left(z_{3}=0\right)$ we have

$$
\dot{z}_{1}=-4, \quad \dot{z}_{2}=-2 z_{2}^{2}, \quad \dot{z}_{3}=0 .
$$


This system has no equilibrium point and its dynamics is given in Figure 4.

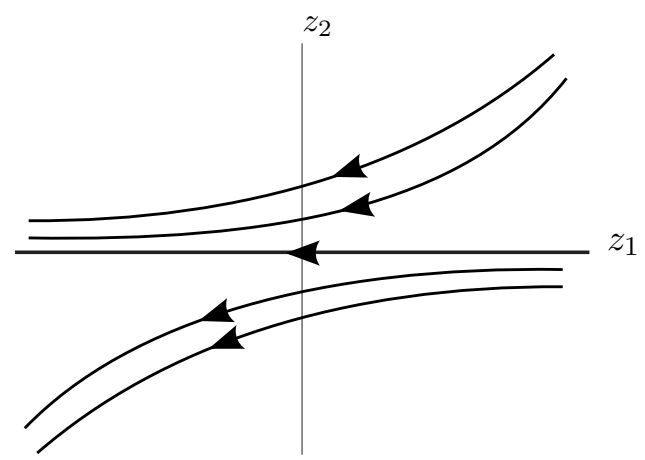

FiguRE 4 . The phase portrait in the local chart $\mathcal{U}_{2}$ at infinity.

Note that in this figure the invariant line $z_{2}=0$ is associated to half of the equator of the Poincaré-Lyapunov sphere that is located at the end of the halfplane $\pi_{+}=\{(x, y, 0), y \geq 0\}$.

3.3. Local chart $\mathcal{U}_{3}$. In the local chart $\mathcal{U}_{3}$ the Poincaré-Lyapunov compactification of the Benoît system is given by

$$
\dot{z}_{1}=z_{1}-2 z_{2}+z_{1}^{2} z_{3}, \quad \dot{z}_{2}=2 z_{2}\left(1+z_{1} z_{3}\right)+z_{3}^{3}, \quad \dot{z}_{3}=z_{3}\left(1+z_{1} z_{3}\right) .
$$

Restricted to $z_{3}=0$ we obtain

$$
\dot{z}_{1}=z_{1}-2 z_{2}, \quad \dot{z}_{2}=2 z_{2}, \quad \dot{z}_{3}=0 .
$$

So this system has a unique equilibrium point in $z_{3}=0$ at the origin of the local chart $\mathcal{U}_{3}$. Moreover, restricted to the invariant set $z_{3}=0$ we have a hyperbolic node that is unstable. Its phase portrait restrict to $z_{3}=0$ is given in Figure 5 .

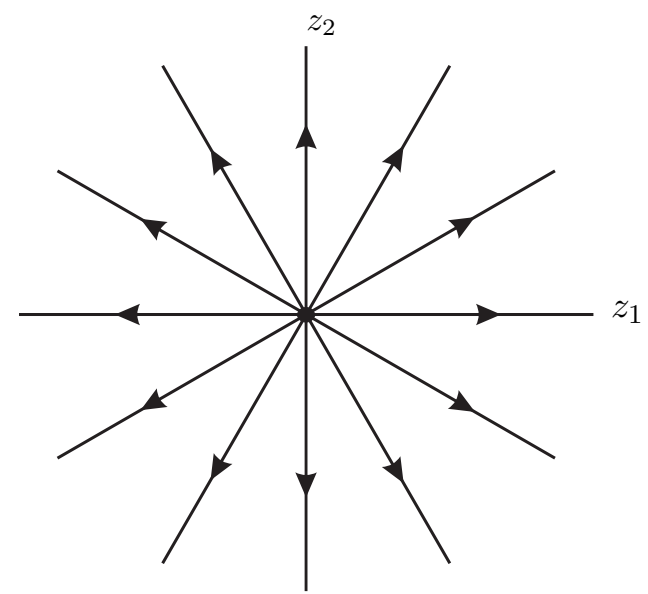

Figure 5. The local phase portrait at the origin of the local chart $\mathcal{U}_{3}$ restricted to $\mathbb{S}^{2}$. 
Remark 6. We observe that the flows in the $\mathcal{V}_{i}$ charts for $i=1,2,3$ are the same than the ones in the respective $\mathcal{U}_{i}$ charts for $i=1,2,3$ but reversing the time because the compactified vector field in $\mathcal{V}_{i}$ coincides with the vector field in $\mathcal{U}_{i}$ multiplied by -1 for each $i=1,2,3$.

From the subsections 3.1-3.3 it follows the proof of Proposition 1.

\section{First Integrals of Benoît system, general solution and local DYNAMICS INSIDE THE LEVELS OF $H_{1}$}

In this section we will prove Proposition 2 that shows that the Benoît system admits two independent first integrals. Moreover we prove Proposition 3 and Corollary 4 where we give an explicit form of the general solution of the Benoit system. We also study the local phase portrait at the equilibrium point on the compactified levels of the first integral $H_{1}$.

Proof of Proposition 2. First dividing the first and third equation of the Benoitt system by the second one we obtain the equivalent system

$$
\frac{d x}{d y}=-2 y, \quad \frac{d z}{d y}=-x-z^{2} .
$$

Solving this new system we obtain

$$
x(y)=-y^{2}+H_{1},
$$

$$
\begin{aligned}
z(y)= & -\frac{2 H_{2} W_{1}\left(\frac{H_{1}}{4}+1, \frac{1}{4}, y^{2}\right)}{y\left(H_{2} W_{1}\left(\frac{H_{1}}{4}, \frac{1}{4}, y^{2}\right)+W_{2}\left(\frac{H_{1}}{4}, \frac{1}{4}, y^{2}\right)\right)}-\frac{\left(H_{2}-2 H_{2} y^{2}+H_{1} H_{2}\right) W_{1}\left(\frac{H_{1}}{4}, \frac{1}{4}, y^{2}\right)}{2 y\left(H_{2} W_{1}\left(\frac{H_{1}}{4}, \frac{1}{4}, y^{2}\right)+W_{2}\left(\frac{H_{1}}{4}, \frac{1}{4}, y^{2}\right)\right)} \\
& +\frac{(3+H 1) W_{2}\left(\frac{H_{1}}{4}+1, \frac{1}{4}, y^{2}\right)}{2 y\left(H_{2} W_{1}\left(\frac{H_{1}}{4}, \frac{1}{4}, y^{2}\right)+W_{2}\left(\frac{H_{1}}{4}, \frac{1}{4}, y^{2}\right)\right)}-\frac{\left(1-2 y^{2}+H 1\right) W_{2}\left(\frac{H_{1}}{4}, \frac{1}{4}, y^{2}\right)}{2 y\left(H_{2} W_{1}\left(\frac{H_{1}}{4}, \frac{1}{4}, y^{2}\right)+W_{2}\left(\frac{H_{1}}{4}, \frac{1}{4}, y^{2}\right)\right)} .
\end{aligned}
$$

Solving the above system with respect to $H_{1}$ and $H_{2}$ we obtain the two first integrals given in the statement of Proposition 2. Since their gradients are linearly independent except in a Lebesgue measure set of points of $\mathbb{R}^{3}$, these two first integrals are independent.

Proof of Proposition 3. Solving the first and second equation of the Benoit system with initial conditions $x(0)=x_{0}$ and $y(0)=y_{0}$, we get $x(t)=-t^{2}-y_{0} t+x_{0}$ and $y(t)=t+y_{0}$.

Substituting these expressions into the third equation of the Benoît system we obtain $\dot{z}=t^{2}+y_{0} t-x_{0}-z^{2}$. Now solving this Riccati differential equation with initial condition $z(0)=z_{0}$, we obtain the expression given in the statement of Proposition 3.

Before proving Corollary 4 we give a general definition of the $\alpha$ - and $\omega$-limit set. It is well known that any solution $\varphi(t)=(x(t), y(t), z(t))$ given in Proposition 3 has a maximal interval of definition $(\alpha, \omega)$ with $-\infty \leq \alpha<0$ and $0<\omega \leq+\infty$. We say that a point $y$ is in the $\alpha$-limit set of the orbit $\varphi(t)$ if there exists a strictly decreasing sequence of times $\left(t_{n}\right)_{n \in \mathbb{N}} \subset(\alpha, \omega)$ such that $t_{n} \rightarrow \alpha$ when $n \rightarrow \infty$ and $\varphi\left(t_{n}\right) \rightarrow y$. In this case we denote $y \in \mathcal{L}_{\alpha}(\varphi)$, and call $\mathcal{L}_{\alpha}(\varphi)$ the $\alpha$-limit set of the orbit $\varphi(t)$. In the same way we say that a point $y$ is in the $\omega$-limit set of the orbit $\varphi(t)$ if there exists a strictly increasing sequence of times $\left(t_{n}\right)_{n \in \mathbb{N}} \subset(\alpha, \omega)$ 
such that $t_{n} \rightarrow \omega$ when $n \rightarrow \infty$ and $\varphi\left(t_{n}\right) \rightarrow y$. In this case we denote $y \in \mathcal{L}_{\omega}(\varphi)$, and call $\mathcal{L}_{\omega}(\varphi)$ the $\omega$-limit set of the orbit $\varphi(t)$.

Remark 7. It is known (see Theorem 2.1 of [7]) that when in the maximal interval of definition $\alpha>-\infty$ (resp. $\omega<+\infty)$, then the solution $\varphi(t)$ goes to the boundary of the phase space when $t \rightarrow \alpha$ (resp. when $t \rightarrow \omega)$. In this case all the point in $\mathcal{L}_{\alpha}(\varphi)$ (resp. $\left.\mathcal{L}_{\omega}(\varphi)\right)$ are in the boundary of the phase space.

Proof of Corollary 4. Note that if the solution $\varphi(t)=(x(t), y(t), z(t))$ given in Proposition 3 is such that its maximal interval of definition $(\alpha, \omega)$ has $\omega=+\infty$, it follows from the first and second coordinates of the solution $\varphi(t)$ that $\varphi(t)$ is unbounded in forward time. On the other hand if $\omega<+\infty$ it implies from Remark 7 that the solution goes to the boundary of the phase space when $t \rightarrow \omega$. But as the phase portrait of the Benoît system is the whole $\mathbb{R}^{3}$ it follows that also in this case the solution is unbounded in forward time. Of course the same argument can be applied in backward time and we can conclude that all the solutions of the Benoit system are unbounded in forward and backward times.

The next result describes the geometry of the level sets of the first integral $H_{1}$ in $\mathbb{R}^{3}$ and at infinity.

Proposition 8. For each $h_{1} \in \mathbb{R}$ the level surface $H_{1}=h_{1}$ is a parabolic cylinder in $\mathbb{R}^{3}$. Moreover these parabolic cylinders reach the infinity in the half great circle of the Poincaré sphere that is located at the infinity of the half-plane $\hat{\pi}_{-}=\{(x, 0, z) ; x \leq$ $0\}$.

Proof. It is easy to see that for each $h_{1} \in \mathbb{R}$ the surface $H_{1}=h_{1}$ is a parabolic cylinder in $\mathbb{R}^{3}$. To see how these cylinders arrive at infinity we have to see the form of these cylinders in the local charts $\mathcal{V}_{1}, \mathcal{U}_{3}$ and $\mathcal{V}_{3}$.

In the local chart $\mathcal{V}_{1}$ the level surface $H_{1}=h_{1}$ is given by $z_{3}^{2}+z_{1}^{2}-h_{1} z_{3}^{4}=0$. So at infinity $\left(z_{3}=0\right)$ we have $z_{1}^{2}=0$. So we obtain points of the form $\left(0, z_{2}, 0\right)$ that at infinity in this local chart correspond to the end of the half-plane $\hat{\pi}_{-}=$ $\{(x, 0, z), x \leq 0\}$.

Now in the local chart $\mathcal{U}_{3}$ we have $z_{1} z_{3}^{3}+z_{2}^{2}-h_{1} z_{3}^{4}=0$ that at infinity becomes $z_{2}=0$. So the parabolic cylinder $x+y^{2}=h_{1}$ at infinity in $\mathcal{U}_{3}$ is contained in the straight line $\left(z_{1}, 0,0\right)$. In this way the parallel lines contained in the parabolic cylinders reach the infinity in the same point, the origin of the local chart $\mathcal{U}_{3}$.

The analysis in the local chart $\mathcal{V}_{3}$ follows in a similar way.

The dynamics of the Benoît system restricted to the levels of the $H_{1}$ first integral will be provided studying the local behavior around the equilibria that are in each level. For this purpose we will use the next theorem that describes the topological type of degenerated singularities that have a single non-zero eigenvalue in the plane. This kind of singularities will be called semi-hyperbolic singularities. A proof of this result can be found in Theorem 2.19 of [6].

Theorem 9. Let $(0,0)$ be an isolated singularity of the system

$$
\begin{aligned}
& \dot{x}=X_{1}(x, y), \\
& \dot{y}=y+X_{2}(x, y),
\end{aligned}
$$

where $X_{1}$ and $X_{2}$ are analytic in a neighborhood of the origin and have expansions that begin with second degree terms in $x$ and $y$. Let $y=f(x)$ be the solution of the 
equation $y+X_{2}(x, y)=0$ in the neighborhood of $(0,0)$, and assume that the series expansion of the function $g(x)=X_{1}(x, f(x))$ has the form $g(x)=a_{m} x^{m}+\ldots$, where $m \geq 2, a_{m} \neq 0$. Then

(i) If $m$ is odd and $a_{m}>0$, then $(0,0)$ is a topological node.

(ii) If $m$ is odd and $a_{m}<0$, then $(0,0)$ is a topological saddle, two of whose separatrices tend to $(0,0)$ in the directions 0 and $\pi$, the other two in the directions $\pi / 2$ and $3 \pi / 2$.

(iii) If $m$ is even, then $(0,0)$ is a saddle-node, i.e. a singularity whose neighborhood is the union of one parabolic and two hyperbolic sectors, two of whose separatrices tend to $(0,0)$ in the directions $\pi / 2$ and $3 \pi / 2$ and the other in the direction 0 and $\pi$ according to $a_{m}<0$ or $a_{m}>0$.

The corresponding indices are $+1,-1,0$ so they may serve to distinguish the three types.

Going back to the Benoît system observe that for each $h_{1}$ the surface $H_{1}=h_{1}$ has in the Poincaré sphere three singular points where two of them are hyperbolic nodes (the equilibria $(0,0,1) \in \mathbb{S}^{2}$ and $\left.(0,0,-1) \in \mathbb{S}^{2}\right)$ and one is semi-hyperbolic (the equilibrium $\left.(-1,0,0) \in \mathbb{S}^{2}\right)$.

Now we have to study the local dynamics of the semi-hyperbolic singularity at the equator. This dynamics will be provided by the next result.

Proposition 10. For each fixed $h_{1}$ the semi-hyperbolic singularity $(-1,0,0) \mathbb{S}^{2}$ in the level $H_{1}=h_{1}$ has two nodal sectors and two hyperbolic sectors. More precisely the local phase portrait at $(-1,0,0) \in \mathbb{S}^{2}$ and restricted to $H_{1}=h_{1}$ is described in Figure 6 .

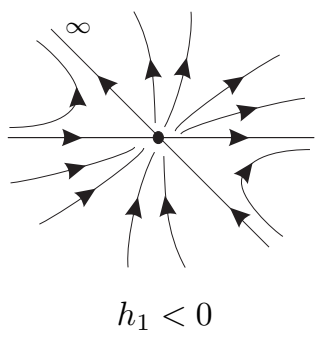

(a)

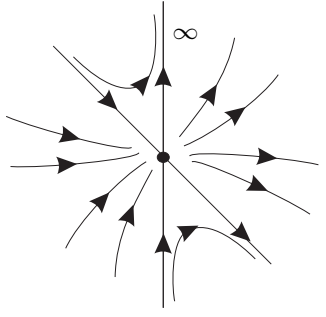

$h_{1}=0$

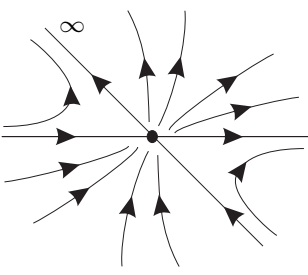

$h_{1}>0$

$(c)$

Figure 6. Local phase portrait at the equilibrium point $(-1,0,0) \in \mathbb{S}^{2}$ on the level $H_{1}=h_{1}$. In the picture we have indicated with the symbol $\infty$ the local invariant straight line of the parabolic cylinder at infinity. Picture (b) is the origin in coordinates $z_{1}, z_{2}$ and picture (a) (resp. (c)) is the origin in coordinates $z_{2}, \theta$ see (14) (resp. (10)).

Proof. In order to study the local dynamics in a neighbourhood of $(-1,0,0) \in \mathbb{S}^{2}$ on the level $H_{1}=h_{1}$ we consider the local chart $\mathcal{V}_{1}$. In this chart we take the 
coordinates given by the Poincaré compactification

$$
x=\frac{1}{z_{3}}, \quad y=\frac{z_{1}}{z_{3}}, \quad z=\frac{z_{2}}{z_{3}} .
$$

Observe that the dynamics at the end of the half-plane $\hat{\pi}_{-}=\{(x, 0, z) ; x \leq$ $0\}$ in these coordinates is the same one obtained using the Poincaré-Lyapunov compactification. So $H_{1}$ takes the form

$$
z_{3}+z_{1}^{2}-h_{1} z_{3}^{2}=0
$$

Moreover the dynamics in the local chart $\mathcal{V}_{1}$ is given by

$$
\begin{array}{ll}
\dot{z}_{1}=-z_{3}\left(2 z_{1}^{2}+z_{3}\right) & =f_{1}\left(z_{1}, z_{2}, z_{3}\right), \\
\dot{z}_{2}=z_{2}^{2}+z_{3}-2 z_{1} z_{2} z_{3} & =f_{2}\left(z_{1}, z_{2}, z_{3}\right), \\
\dot{z}_{3}=-2 z_{1} z_{3}^{2} & =f_{3}\left(z_{1}, z_{2}, z_{3}\right) .
\end{array}
$$

Note that the equilibrium at the infinity $(-1,0,0) \in \mathbb{S}^{2}$ is represented in this local chart by $(0,0,0)$.

Now we will divide the study in three cases depending on $h_{1}$.

Case 1: $h_{1}=0$ (parabolic cylinder). In this case (4) takes the form $z_{3}+z_{1}^{2}=0$. Substituting $z_{3}=-z_{1}^{2}$ in (5) we obtain that the dynamics in the level $H_{1}=0$ is given by

$$
\begin{aligned}
& \dot{z}_{1}=f_{1}\left(z_{1}, z_{2},-z_{1}^{2}\right)=z_{1}^{4}, \\
& \dot{z}_{2}=f_{2}\left(z_{1}, z_{2},-z_{1}^{2}\right)=z_{2}^{2}-z_{1}^{2}+2 z_{1}^{3} z_{2} .
\end{aligned}
$$

In this differential system the origin $(0,0)$ has the eigenvalue 0 with multiplicity 2. In order to understand the behavior of the flow of (6) in a neighborhood of $(0,0)$ we will use a polar blow-up. So doing the change of variables $z_{1}=r_{1} \cos \theta_{1}$, $z_{2}=r_{1} \sin \theta_{1}$ and the rescaling of the time $s=r_{1} t$, system (6) becomes

$$
\begin{aligned}
& r_{1}^{\prime}=r_{1}\left(r_{1}^{2} \cos ^{5} \theta_{1}+2 r_{1}^{2} \sin ^{2} \theta_{1} \cos ^{3} \theta_{1}-\sin \theta_{1} \cos ^{2} \theta_{1}+\sin ^{3} \theta_{1}\right), \\
& \theta_{1}^{\prime}=\cos \theta_{1}\left(r_{1}^{2} \sin \theta_{1} \cos ^{3} \theta_{1}-\cos ^{2} \theta_{1}+\sin ^{2} \theta_{1}\right),
\end{aligned}
$$

where the prime denotes the derivative with respect to the new time $s$.

Now we study the zeroes of (7) restricted to $r_{1}=0$. For this we solve $\theta_{1}^{\prime}=0$, $r_{1}=0$ that implies that

$$
\theta_{1}= \pm \frac{\pi}{4}, \pm \frac{\pi}{2}, \pm \frac{3 \pi}{4}
$$

We must analyze the topological type of each one of these singularities.

Calling $X$ the vector field (7) we have for $\theta_{1}^{*}=\frac{\pi}{4}$

$$
D X\left(0, \frac{\pi}{4}\right)=\left(\begin{array}{ll}
0 & 0 \\
0 & \sqrt{2}
\end{array}\right) .
$$

So $\left(0, \frac{\pi}{4}\right)$ is a semi-hyperbolic singularity of $(7)$ and to decide about its topological type we use Theorem 9 .

By a translation of the form $R_{1}=r_{1}$ and $\phi_{1}=\theta_{1}-\frac{\pi}{4}$ and a time reparametrization $\tau=\sqrt{2} s$ we obtain 


$$
\begin{aligned}
\dot{R}_{1}= & \frac{1}{4}\left(\cos \phi_{1}-\cos \left(3 \phi_{1}\right)+\sin \phi_{1}+\sin \left(3 \phi_{1}\right)\right) R_{1}+ \\
& \frac{\sqrt{2}}{2}\left(\cos ^{5}\left(\phi_{1}+\frac{\pi}{4}\right)+2 \sin ^{2}\left(\phi_{1}+\frac{\pi}{4}\right) \cos ^{3}\left(\phi_{1}+\frac{\pi}{4}\right)\right) R_{1}^{3}+O\left(R_{1}^{4}\right)=X_{1}\left(R_{1}, \phi_{1}\right), \\
\dot{\phi}_{1}= & \frac{1}{4}\left(-\cos \phi_{1}+\cos \left(3 \phi_{1}\right)+\sin \phi_{1}+\sin \left(3 \phi_{1}\right)\right) \\
& \frac{1}{\sqrt{2}} \cos ^{4}\left(\phi_{1}+\frac{\pi}{4}\right) \sin \left(\phi_{1}+\frac{\pi}{4}\right) R_{1}^{2}+O\left(R_{1}^{4}\right)=\tilde{X}_{2}\left(R_{1}, \phi_{1}\right),
\end{aligned}
$$

and the dots denotes derivative with respect to $\tau$.

Expanding $\tilde{X}_{2}\left(R_{1}, \phi_{1}\right)$ in Taylor series of $\phi_{1}$ around 0 we obtain $\tilde{X}_{2}\left(R_{1}, \phi_{1}\right)=$ $\phi_{1}+X_{2}\left(R_{1}, \phi_{1}\right)$, where $X_{2}$ has terms of second order in $R_{1}$ and $\phi_{1}$. Solving $\phi_{1}+$ $X_{2}\left(R_{1}, \phi_{1}\right)=0$ for $\phi_{1}$ as a function of $R_{1}$ in a neighborhood of 0 we obtain

$$
\phi_{1}=-\frac{1}{8} R_{1}^{2}+O\left(R_{1}^{4}\right)=f\left(R_{1}\right) .
$$

Substituting in $X_{1}\left(R_{1}, \phi_{1}\right)$ we have

$$
g\left(R_{1}\right)=X_{1}\left(R_{1}, f\left(R_{1}\right)\right)=\frac{1}{4} R_{1}^{3}+O\left(R_{1}^{4}\right) .
$$

Using the notation of Theorem 9 we have that $m=3$, and $a_{3}=\frac{1}{4}>0$. It follows from Theorem $9(\mathrm{i})$ that $(0,0)$ is a topological node.

Repeating this procedure for the equilibrium $\left(0,-\frac{\pi}{4}\right)$, also semi-hyperbolic, we get

$$
g\left(R_{1}\right)=-\frac{1}{4} R_{1}^{3}+O\left(R_{1}^{4}\right) .
$$

Then Theorem 9 implies that the equilibrium is a topological saddle.

Consider now the equilibria $\left(0, \pm \frac{\pi}{2}\right)$. For these equilibria the Jacobian matrix satisfies

$$
D X\left(0, \pm \frac{\pi}{2}\right)=\left(\begin{array}{cc} 
\pm 1 & 0 \\
0 & \mp 1
\end{array}\right) \text {. }
$$

So these two equilibria are hyperbolic saddles with expanding (resp. contracting) radial direction for $\left(0, \frac{\pi}{2}\right)$ (resp. $\left.\left(0,-\frac{\pi}{2}\right)\right)$.

Taking into account the equilibria $\left(0, \pm \frac{3 \pi}{4}\right)$ we have

$$
D X\left(0, \pm \frac{3 \pi}{4}\right)=\left(\begin{array}{ll}
0 & 0 \\
0 & \pm \sqrt{2}
\end{array}\right)
$$

and so both equilibria are semi-hyperbolic. Therefore we can use again Theorem 9 for deciding about their topological type.

Similar calculations to the ones done for the equilibrium $\left(0, \frac{\pi}{4}\right)$ show that for $\left(0, \frac{3 \pi}{4}\right)$ the function $g$ is given by $g\left(R_{1}\right)=-\frac{R_{1}^{2}}{8}+O\left(R_{1}^{4}\right)$, and $f\left(R_{1}\right)=-\frac{R_{1}^{3}}{4}+$ $O\left(R_{1}^{4}\right)$, that implies from Theorem 9 that $\left(0, \frac{3 \pi}{4}\right)$ is a topological saddle. Also 
$\left(0,-\frac{3 \pi}{4}\right)$ is such that $g\left(R_{1}\right)=-\frac{1}{8} R_{1}^{2}+O\left(R_{1}^{4}\right)$, and $f\left(R_{1}\right)=\frac{R_{1}^{3}}{4}+O\left(R_{1}^{4}\right)$, and from Theorem 9 it follows that $\left(0,-\frac{3 \pi}{4}\right)$ is a topological node.

Finally for the case $h_{1}=0$ the local phase portrait after a polar blow-up is given in Figure 7.

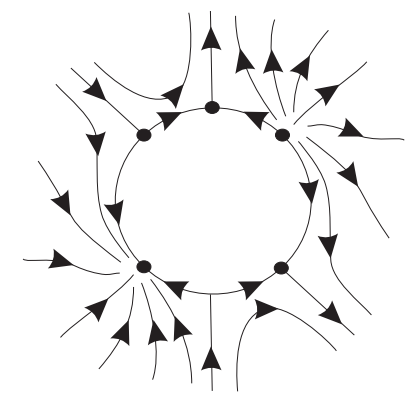

FIgURE 7 . The radial blow-up at the equilibrium $(-1,0,0) \in \mathbb{S}^{2}$ restricted to the level $H_{1}=0$.

This implies that the local phase portrait of system (6) in a neighborhood of $(0,0)$ is given by Figure $6(\mathrm{~b})$.

For the next steps consider $h_{1} \neq 0$. Here we can write (4) as

$$
\frac{z_{1}^{2}}{-\frac{1}{4 h_{1}}}+\frac{\left(z_{3}-\frac{1}{2 h_{1}}\right)^{2}}{\frac{1}{4 h_{1}^{2}}}=1 .
$$

Equation (8) implies that the invariant surfaces $H_{1}=h_{1}$ are represented in the local chart $\mathcal{V}_{1}$ by a hyperbolic cylinder if $h_{1}>0$, or by an elliptic cylinder if $h_{1}<0$.

Case 2: $h_{1}>0$ (hyperbolic cylinder). In this case equation (8) becomes

$$
-\frac{z_{1}^{2}}{a^{2}}+\frac{\left(z_{3}-b\right)^{2}}{b^{2}}=1
$$

where $a=\sqrt{\frac{1}{4 h_{1}}}$ and $b=\frac{1}{2 h_{1}}$.

Performing the change of coordinates

$$
z_{1}=\operatorname{ar} \sinh \theta, \quad z_{2}=z_{2} \quad \text { and } \quad z_{3}=b-b r \cosh \theta
$$

system (5) becomes

$$
\begin{aligned}
\dot{r} & =\frac{b}{a}(r \cosh \theta-1)\left(2 a^{2} r^{2}+\left(b-2 a^{2}\right) r \cosh \theta-b\right) \sinh \theta, \\
\dot{z}_{2} & =z_{2}^{2}+2 a b r z_{2}(r \cosh \theta-1) \sinh \theta+b-b r \cosh \theta \\
\dot{\theta} & =-\frac{b}{2 a r}(r \cosh \theta-1)\left(r\left(2 a^{2}+b+\left(b-2 a^{2}\right) \cosh (2 \theta)\right)-2 b \cosh \theta\right)
\end{aligned}
$$

and (9) is given by $r^{2}=1$.

Observe that the equilibrium $\left(z_{1}, z_{2}, z_{3}\right)=(0,0,0)$ in the local chart $\mathcal{V}_{1}$ now is given, in the new coordinates by $\left(r, z_{2}, \theta\right)=(1,0,0)$. As we want to study the 
dynamics in a neighborhood of $(1,0,0)$ restricted to the level surface $r=1$ we can restrict to the reduced system

$$
\begin{aligned}
& \dot{z}_{2}=z_{2}^{2}+2 a b z_{2}(\cosh \theta-1) \sinh \theta+b-b \cosh \theta, \\
& \dot{\theta}=\frac{4 b}{a}\left(2 a^{2}+\left(2 a^{2}-b\right) \cosh \theta\right) \sinh ^{4}(\theta / 2) .
\end{aligned}
$$

Now we have to investigate the topological type of the equilibrium $(0,0)$ of this system. This equilibrium has all the eigenvalues zero, and so we must use the blow-up technic again. Taking $z_{2}=r_{1} \cos \theta_{1}$ and $\theta=r_{1} \sin \theta_{1}$ the previous system becomes

$(12)$

$$
\begin{aligned}
\dot{r}_{1}= & 8 a b \sin \theta_{1} \sinh ^{4}\left(\frac{r_{1}}{2} \sin \theta_{1}\right) \\
& +\cos \theta_{1}\left(r_{1}^{2} \cos ^{2} \theta_{1}+a b r_{1}\left(\sinh \left(2 r_{1} \sin \theta_{1}\right)-2 \sinh \left(r_{1} \sin \theta_{1}\right)\right) \cos \theta_{1}+b\right) \\
& -\frac{b}{a} \cosh \left(r_{1} \sin \theta_{1}\right)\left(4\left(b-2 a^{2}\right) \sin \theta_{1} \sinh ^{4}\left(\frac{1}{2} r_{1} \sin \theta_{1}\right)+a \cos \theta_{1}\right), \\
\dot{\theta}_{1}= & \frac{4 b}{a r_{1}} \cos \theta_{1}\left(2 a^{2}+\left(2 a^{2}-b\right) \cosh \left(r_{1} \sin \theta_{1}\right)\right) \sinh ^{4}\left(\frac{1}{2} r_{1} \sin \theta_{1}\right)-r_{1} \sin \theta_{1} \cos ^{2}\left(\theta_{1}\right) \\
& -\frac{\sin \theta_{1}}{r_{1}}\left(2 a b r_{1}\left(\cosh \left(r_{1} \sin \theta_{1}\right)-1\right) \sinh \left(r_{1} \sin \theta_{1}\right) \cos \theta_{1}+b-b \cosh \left(r_{1} \sin \theta_{1}\right)\right) .
\end{aligned}
$$

Expanding both equations of system (12) in Taylor series of $r_{1}$ and rescaling $s=r_{1} t$, system 12 writes

$$
\begin{aligned}
& r_{1}^{\prime}=\left(\cos ^{3} \theta_{1}-\frac{b \cos \theta_{1} \sin ^{2} \theta_{1}}{2}\right) r_{1}+O\left(r_{1}^{3}\right) \\
& \theta_{1}^{\prime}=\frac{b \sin ^{3} \theta_{1}-2 \cos ^{2} \theta_{1} \sin \theta_{1}}{2}+\left(\frac{b \sin ^{5} \theta_{1}}{24}-\frac{b^{2} \cos \theta_{1} \sin ^{4} \theta_{1}}{4 a}\right) r_{1}^{2}+O\left(r_{1}^{3}\right),
\end{aligned}
$$

where the prime denotes derivative with respect to the new time $s$.

Now, as in the case $h_{1}=0$, we have to study the zeroes of this system restricted to $r_{1}=0$. Solving $\theta_{1}^{\prime}=0$ and $r_{1}=0$ we get

$$
\theta_{1}=0, \arctan \sqrt{\frac{2}{b}}, \pi-\arctan \sqrt{\frac{2}{b}}, \pi, \pi+\arctan \sqrt{\frac{2}{b}},-\arctan \sqrt{\frac{2}{b}} .
$$

Again we have to study each one of these equilibria separately in order to obtain the dynamics in a neighborhood of $(0,0)$.

We begin with the equilibrium $\left(r_{1}^{*}, \theta_{1}^{*}\right)=(0,0)$ of the previous system. For this equilibrium we have $D X(0,0)=\left(\begin{array}{cc}1 & 0 \\ 0 & -1\end{array}\right)$. So $(0,0)$ is a hyperbolic saddle with expanding radial direction. For $\left(r_{1}^{*}, \theta_{1}^{*}\right)=(0, \pi)$ we obtain $D X(0, \pi)=\left(\begin{array}{cc}-1 & 0 \\ 0 & 1\end{array}\right)$ and this equilibrium is also a hyperbolic saddle but with contracting radial direction.

Take now $\left(r_{1}^{*}, \theta_{1}^{*}\right)=\left(0, \arctan \sqrt{\frac{2}{b}}\right)$. Here $D X\left(0, \arctan \sqrt{\frac{2}{b}}\right)=\left(\begin{array}{cc}0 & 0 \\ 0 & \frac{2}{\sqrt{\frac{2+b}{b}}}\end{array}\right)$. And as in case 1 we have a semi-hyperbolic equilibrium point. So we use one more time Theorem 9 for deciding about the topological type of this singularity. 
By a translation of the form $r_{1}=R_{1}, \theta_{1}=\phi_{1}+\arctan \sqrt{\frac{2}{b}}$ and the time reparametrization $\tau=\sqrt{\frac{2+b}{b}} t$ we find

$$
\begin{aligned}
& R_{1}^{\prime}=X_{1}\left(R_{1}, \phi_{1}\right), \\
& \phi_{1}^{\prime}=\phi_{1}+X_{2}\left(R_{1}, \phi_{2}\right),
\end{aligned}
$$

where

$$
\begin{aligned}
X_{1}\left(R_{1}, \phi_{1}\right)= & -\frac{b}{4} \sqrt{\frac{b+2}{b}} \cos \left(\phi_{1}+\arctan \sqrt{\frac{2}{b}}\right) \sin ^{2}\left(\phi_{1}+\arctan \sqrt{\frac{2}{b}}\right) R_{1} \\
& +\frac{1}{2} \sqrt{\frac{b+2}{b}} \cos ^{3}\left(\phi_{1}+\arctan \sqrt{\frac{2}{b}}\right) R_{1}+O\left(R_{1}^{3}\right),
\end{aligned}
$$

and $\phi_{1}+X_{2}\left(R_{1}, \phi_{2}\right)$ is

$$
\begin{aligned}
& \frac{1}{4} \sqrt{\frac{b+2}{b}}\left(b \sin ^{3}\left(\phi_{1}+\arctan \sqrt{\frac{2}{b}}\right)-2 \cos ^{2}\left(\phi_{1}+\arctan \sqrt{\frac{2}{b}}\right) \sin \left(\phi_{1}+\arctan \sqrt{\frac{2}{b}}\right)\right) \\
& -\frac{b}{48 a} \sqrt{\frac{b+2}{b}} \sin ^{4}\left(\phi_{1}+\arctan \sqrt{\frac{2}{b}}\right)\left(6 b \cos \left(\phi_{1}+\arctan \sqrt{\frac{2}{b}}\right)-a \sin \left(\phi_{1}+\arctan \sqrt{\frac{2}{b}}\right)\right) R_{1}^{2} \\
& +O\left(R_{1}^{4}\right) .
\end{aligned}
$$

Solving $\phi_{1}+X_{2}\left(R_{1}, \phi_{2}\right)=0$ for $\phi_{1}$ as a function of $R_{1}$ in a neighborhood of 0 we have

$$
\begin{aligned}
\phi_{1}= & f\left(R_{1}\right)=-\frac{b(a \sqrt{2} b-6 b)}{12 a(2+b)^{2}} R_{1}^{2}+ \\
& \frac{b\left(-320(b+2) a^{3}+\sqrt{\frac{2}{b}}(9 b-2) a^{2}+40 b(5 b+16) a+30 \sqrt{2}(5 b-2) b^{5 / 2}\right)}{480 a^{2}(b+2)^{4}} R_{1}^{4} \\
& +O\left(R_{1}^{5}\right)
\end{aligned}
$$

and so

$$
g\left(R_{1}\right)=X_{1}\left(R_{1}, f\left(R_{1}\right)\right)=-\frac{b-4 a^{2}}{2 a(b+2) \sqrt{\frac{2}{b}}} R_{1}^{3}+O\left(R_{1}^{4}\right) .
$$

Now observe that $a_{3}=-\frac{\left(b-4 a^{2}\right)}{2 a(b+2) \sqrt{\frac{2}{b}}}>0$ because $h_{1}>0$. So from Theorem 9 $\left(0, \arctan \sqrt{\frac{2}{b}}\right)$ is a topological node.

It is not difficult to see that for the equilibrium $\left(0,-\arctan \sqrt{\frac{2}{b}}\right)$ we have $D X\left(0,-\arctan \sqrt{\frac{2}{b}}\right)=\left(\begin{array}{cc}0 & 0 \\ 0 & \frac{2}{\sqrt{\frac{2+b}{b}}}\end{array}\right)$, and in a similar way we obtain for this equilibrium $g\left(R_{1}\right)=X_{1}\left(R_{1}, f\left(R_{1}\right)\right)=\frac{b-4 a^{2}}{2 a(b+2) \sqrt{\frac{2}{b}}} R_{1}^{3}+O\left(R_{1}^{4}\right)$. From Theorem 9 we get that $\left(0,-\arctan \sqrt{\frac{2}{b}}\right)$ is a topological saddle.

Consider now the equilibrium $\left(0, \pi+\arctan \sqrt{\frac{2}{b}}\right)$. Here we have $D X\left(0, \pi+\arctan \sqrt{\frac{2}{b}}\right)=\left(\begin{array}{cc}0 & 0 \\ 0 & -\frac{2}{\sqrt{\frac{2+b}{b}}}\end{array}\right)$. Similar calculations show that for this 
case we obtain

$$
\begin{aligned}
\phi_{1}= & f\left(R_{1}\right)=-\frac{b(a \sqrt{2} b-6 b)}{12 a(2+b)^{2}} R_{1}^{2} \\
& +\frac{b\left(-320(b+2) a^{3}+\sqrt{\frac{2}{b}}(9 b-2) a^{2}+40 b(5 b+16) a+30 \sqrt{2}(5 b-2) b^{5 / 2}\right)}{480 a^{2}(b+2)^{4}} R_{1}^{4} \\
& +O\left(R_{1}^{5}\right),
\end{aligned}
$$

and

$$
g\left(R_{1}\right)=X_{1}\left(R_{1}, f\left(R_{1}\right)\right)=-\frac{b-4 a^{2}}{2 a(b+2) \sqrt{\frac{2}{b}}} R_{1}^{3}+O\left(R_{1}^{5}\right) .
$$

As $a_{3}=-\frac{b-4 a^{2}}{2 a(b+2) \sqrt{\frac{2}{b}}}>0$ for $h_{1}>0$ it follows from Theorem 9 that the equilib$\operatorname{rium}\left(0, \pi+\arctan \sqrt{\frac{2}{b}}\right)$ is a topological node. In the same way we can show that the equilibrium $\left(0, \pi-\arctan \sqrt{\frac{2}{b}}\right)$ is a topological saddle.

All together for the case $h_{1}>0$ the local phase portrait after a polar blow-up is given in Figure 8.

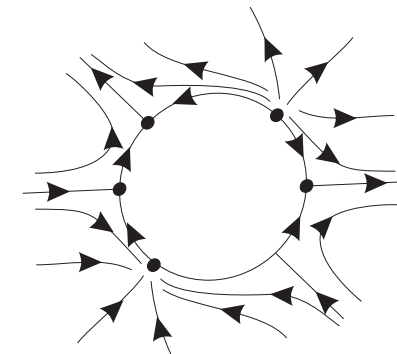

FiguRE 8 . The radial blow-up at the equilibrium $(-1,0,0) \in \mathbb{S}^{2}$ restricted to the level $H_{1}=h_{1}>0$.

So the dynamics restricted to the level $H_{1}=h_{1}$ in a neighborhood of the equilibrium at infinity $(-1,0,0) \in \mathbb{S}^{2}$ is given by Figure $6(\mathrm{c})$.

Case 3: $h_{1}<0$ (elliptic cylinder). In this case equation (8) becomes

$$
\frac{z_{1}^{2}}{a^{2}}+\frac{\left(z_{3}-b\right)^{2}}{b^{2}}=1 .
$$

Here performing a change of coordinates of the form

$$
z_{1}=\operatorname{arcos} \theta, \quad z_{2}=z_{2} \quad \text { and } \quad z_{3}=b+b r \sin \theta
$$

we obtain a system very near to the system studied in case 2 . The proof for this case is very close to the proof of case 2 and will be omitted.

From the above proposition we are in condition to prove Theorem 5. This will be done in the next section. 


\section{Proof of Theorem 5}

The proof of Theorem 5 will be divided into two parts. First we prove that all the possible configurations of the phase portrait restricted to the levels of the first integral $H_{1}$ are the ones given in Figure 2. In the second step we provide solutions of the Benoit system that show that the three possible cases in fact occur at different levels of $H_{1}$.

Proof of the first part of Theorem 5. By the Poincaré-Bendixon theorem (see for instance [6]) at the compactified level $H_{1}=h_{1}$ inside the Poincaré ball, the only possible $\alpha$ - and $\omega$-limit sets of any orbit of the level set $H_{1}=h_{1}$ is an equilibrium point. Note that at $H_{1}=h_{1}$ there are exactly 3 equilibrium points which are the northern and southern poles that are hyperbolic nodes and the semi-hyperbolic equilibrium at $(-1,0,0) \in \mathbb{S}^{2}$ which local dynamics is given by Proposition 10 .

From the local dynamics of $(-1,0,0) \in \mathbb{S}^{2}$ (see Proposition 6 ) in the level $H_{1}=$ $h_{1}$ we note that there are two nodal zones with one attracting and other repeller. So if an orbit visits both nodal zones this orbit is homoclinic for the equilibrium $(-1,0,0) \in \mathbb{S}^{2}$, being an orbit of disconnecting type. Moreover in this case we can have a unique homoclinic orbit to $(-1,0,0) \in \mathbb{S}^{2}$ (Figure $2(\mathrm{~b})$ ) or a continuous of homoclinic orbits to $(-1,0,0) \in \mathbb{S}^{2}$ (Figure $2(\mathrm{a})$ ). Note that the existence of two homoclinic orbits to $(-1,0,0) \in \mathbb{S}^{2}$ in a same level $H_{1}=h_{1}$ implies the existence of a continuum of such orbits. Note that if such an orbit exists it disconnects the cylinder $H_{1}=h_{1}$ into two connected components being one containing the northern pole and the other containing the southern pole. In this case all the orbits in the connected component that contains the northern pole are such that their $\omega$-limit set is the equilibrium $(-1,0,0) \in \mathbb{S}^{2}$, and their $\alpha$-limit set is either the northern pole and we have an heteroclinic orbits connecting the norther pole and the equilibrium $(0,0,1)$ (orbit of intermediate type), or the $\alpha$-limit set is the equilibrium $(-1,0,0)$ and we have a homoclinic orbit to $(-1,0,0) \in \mathbb{S}^{2}$ (orbit of disconnecting type).

Similar analysis can be done for the orbits in the region that contain the southern pole and again we obtain orbits of intermediate type or disconnecting type.

Now if the Benoit system does not admit homoclinic orbits for the equilibrium $(-1,0,0)$ at the level $H_{1}=h_{1}$ then all the orbits that have the northern pole as $\alpha$-limit set can have either $(-1,0,0)$ as $\omega$-limit and we obtain an orbit of intermediate type, or the southern pole as $\omega$-limit and in this case we obtain an orbit of passage type.

The next proposition provide an example where all the three different phase portraits for the levels $H_{1}=h_{1}$ are present in a Benoit system. So we obtain the second part of Theorem 5 .

Proposition 11. The Benoît system realizes the three different phase portraits for the levels $H_{1}=h_{1}$ described in Figure 2.

Proof. Solving the Benoît system with initial condition $\left(0,0, z_{0}\right)$ we obtain

$$
\varphi\left(t, z_{0}\right)=(x(t), y(t), z(t))=\left(-t^{2}, t, z\left(t, z_{0}\right)\right)
$$

with 


$$
z\left(t, z_{0}\right)= \begin{cases}\frac{\frac{\left(-\sqrt{2} \pi t \Gamma\left(\frac{3}{4}\right)^{2}+\pi^{2} z_{0} t\right) J_{1}\left(-\frac{3}{4}, \frac{t^{2}}{2}\right)}{\Gamma\left(\frac{3}{4}\right)^{2}}+2 t J_{2}\left(\frac{3}{4}, \frac{t^{2}}{2}\right)}{\frac{\left(-\sqrt{2} \pi \Gamma\left(\frac{3}{4}\right)^{2}+\pi^{2} z_{0}\right) J_{1}\left(\frac{1}{4}, \frac{t^{2}}{2}\right)}{\Gamma\left(\frac{3}{4}\right)^{2}}-2 J_{2}\left(\frac{1}{4}, \frac{t^{2}}{2}\right)} & \text { if } t<0, \\ z_{0} & \text { if } t=0, \\ \frac{\left(\sqrt{2} \pi t \Gamma\left(\frac{3}{4}\right)^{2}+\pi^{2} z_{0} t\right) J_{1}\left(-\frac{3}{4}, \frac{t^{2}}{2}\right)}{\Gamma\left(-\frac{3}{4}\right)^{2}}-2 t J_{2}\left(\frac{3}{4}, \frac{t^{2}}{2}\right) & \text { if } t>0, \\ \frac{\left(\sqrt{2} \pi \Gamma\left(\frac{3}{4}\right)^{2}+\pi^{2} z_{0}\right) J_{1}\left(\frac{1}{4}, \frac{t^{2}}{2}\right)}{\Gamma\left(\frac{3}{4}\right)^{2}}-2 J+2\left(\frac{1}{4}, \frac{t^{2}}{2}\right) & \end{cases}
$$

where the function $\Gamma(\nu)$ is the Gamma and the functions $J_{1}(\nu, s)$ and $J_{2}(\nu, s)$ are the modified Bessel functions of the first end second kinds, respectively. They satisfy the Bessel equation $s^{2} \ddot{y}+s \dot{y}+\left(s^{2}+\nu^{2}\right) y=0$ (see for more detail [1]). Moreover for this solution we get $\lim _{t \rightarrow \pm \infty} z\left(t, z_{0}\right) / x(t)=\lim _{t \rightarrow \pm \infty}-z\left(t, z_{0}\right) / t^{2}=0$. This implies that in the local chart $\mathcal{V}_{1}$ this solution satisfies $z_{1}(t)=y(t) / x(t) \rightarrow 0$ when $t \rightarrow \pm \infty$, and $z_{2}(t)=z\left(t, z_{0}\right) / x(t) \rightarrow 0$ when $t \rightarrow \pm \infty$. Observing that the singularity at infinity $(-1,0,0) \in \mathbb{S}^{2}$ is represented in the local chart $\mathcal{V}_{1}$ by the origin $(0,0,0)$, it follows that this solution is homoclinic to $(-1,0,0) \in \mathbb{S}^{2}$ for each $z_{0} \in \mathbb{R}$. In this way we have proved that the Benoît system has a phase portrait like Figure 2(a) at the level $H_{1}=0$ (note that the solutions (15) are inside the level $\left.H_{1}=0\right)$.

On the other hand, solving the Benoît system with initial condition $\left(x_{0}, y_{0}, z_{0}\right)=$ $(2,0,0)$ we obtain

$$
\varphi\left(t, x_{0}\right)=\left(x\left(t, x_{0}\right), y(t), z\left(t, x_{0}\right)\right)=\left(-t^{2}+2, t, z(t)\right)
$$

where $z(t)$ is

$$
\frac{\left(1-t^{2}\right) \pi J_{1}\left(-\frac{1}{4},-\frac{t^{2}}{2}\right)+\left(-1+t^{2}\right) \sqrt{2} J_{2}\left(\frac{1}{4},-\frac{t^{2}}{2}\right)-t^{2}\left(\sqrt{2} J_{2}\left(\frac{3}{4},-\frac{t^{2}}{2}\right)+\pi J_{1}\left(\frac{3}{4},-\frac{t^{2}}{2}\right)\right)}{t\left(-\sqrt{2} J_{2}\left(\frac{1}{4},-\frac{t^{2}}{2}\right)+\sqrt{2} J_{2}\left(\frac{3}{4},-\frac{t^{2}}{2}\right)+\pi J_{1}\left(-\frac{1}{4},-\frac{t^{2}}{2}\right)+\pi J_{1}\left(\frac{3}{4},-\frac{t^{2}}{2}\right)\right)}
$$

The denominator of this function has zeroes in $t_{-} \approx-1.162342261$ and $t_{+} \approx$ 1.16234226158 moreover $\lim _{t_{\searrow t_{-}}} z(t, 2)=\infty$ and $\lim _{t \nearrow t_{+}} z(t, 2)=-\infty$. This implies that this solution is a heteroclinic solution connecting the point $(0,0,1) \in \mathbb{S}^{2}$ and $(0,0,-1) \in \mathbb{S}^{2}$. So the Benoît system has a phase portrait like Figure 2(c) at the level $H_{1}=2$ (solution (16) is inside the level $H_{1}=2$ ).

These two particular solutions show that the Benoit system admits the three possible phase portraits given in Figure 2.

Remark 12. It is not difficult to see that for the system in Proposition 11 a bifurcation in the phase portrait occurs for the value of the parameter $h_{1}=1$. In fact for $h_{1}=1$ we have a unique homoclinic solution for the equilibrium point $(-1,0,0) \in \mathbb{S}^{2}$ given by $\varphi(t)=\left(-t^{2}+1, t,-t\right)$ and for $h_{1}=1+\delta$ for $\delta>0$ sufficiently small there is no homoclinic orbit at this level. 


\section{ACKNOWLEDGMENTS}

We thank to the referees their comments and suggestions which allow us to improve the presentation of the results of this work.

The first author was partially supported by CNPq grant number 200293/2010-9 and Fapesp grant number 2012/12025-5. The second author is partially supported by MICINN/FEDER grant MTM2008-03437, AGAUR grant number 2009SGR410, by ICREA Academia and by FP7-PEOPLE-2012-IRSES-316338. Both authors are also supported by the joint project CAPES-MECD grant PHB-2009-0025-PC.

\section{REFERENCES}

[1] M. Abramowitz And I.A. Stegun, Handbook of Mathematical Functions. National Bureal of Standards Applied Mathematics, Series 55, 1972.

[2] E. BEnô̂T, Systèmes lents-rapides dans $\mathbb{R}^{3}$ et leurs canards. Astérisque 109-110, (1983), 159-191.

[3] E. BENô̂T, Perturbation singulière en dimension trois: Canards en un pseudo-singulier noeud. Bulletin de la Société Mathématique de France 129-1, (2001), 91-113.

[4] E. Benoît, J.F. Callot, F. Diener And M. Diener, Chasse au canard. Collectanea Mathematica, 31-32 (1-3), (1981), 37-119.

[5] A. Cima And J. Llibre, Bounded polynomial vector fields. Trans. Amer. Math. Soc. 318, (1990), 557-579.

[6] F. Dumortier, J. Llibre And J.C. Artés, Qualitative theory of planar differential systems. Universitext, Springer-Verlag, 2006.

[7] J. HALE, Ordinary Differential Equations. Robert E. Krieger Publishing Company, INC, 1980 .

[8] E.F. Mishchnko, Yu. S. Kolesov, A. Yu. Kolesov and N. Kh. Rhozov, Asymptotic methods in singularly perturbed systems. Monographs in Contemporary Mathematics; Consultants Bureau, New Yord, A Division of Plenum Publishing Cooperation 233 Springer Street, New York, N.Y. 10013, 1994.

[9] P. Szmolyan and M. Wechselberger, Canards in $\mathbb{R}^{3}$. J. Diff. Eq., 177, (2001), 419-453.

[10] M. Wechselberger, Existence and Bifurcation of Canards in $\mathbb{R}^{3}$ in the case of a Folded Node. SIAM J. App. Dyn. Sys., 4, (2005), 101-139.

1 Centro de Matemática ComputaÇão e CogniÇão. Universidade Federal do abC, 09210-170. SANTO AndRÉ. S.P. BRAZIL

E-mail address: mauricio.lima@ufabc.edu.br

2 Departament de Matemàtiques. Universitat Autònoma de Barcelona, 08193 Bellaterra. Barcelona, CAtalonia, Spain

E-mail address: jllibre@mat.uab.cat 